\title{
LA MECANIZACIÓN DEL CAMPO EN LAS ISLAS BALEARES (1960-1970). EL TRÁNSITO DE LA AGRICULTURA TRADICIONAL A LA INDUSTRIAL
}

\author{
Jaume Binimelis Sebastián \\ Antoni Ordinas Garau \\ Departament de Ciències de la Terra. Universitat de les illes Balears \\ jaume.binimelis@uib.es, antoni.ordinas@uib.es
}

\section{RESUMEN}

Se estudia el proceso de modernización de la agricultura balear a través del análisis de la mecanización. El trabajo se centra en la década de los años sesenta y utiliza el Registro de Tractores, fuente específica para el estudio de la mecanización no exenta de inconvenientes, pero de gran utilidad. En 1970, cuando finaliza el período, la motorización ha alcanzado la totalidad del territorio, multiplicándose casi por 10 la potencia del parque de maquinaria agrícola en relación a la existente en 1950.

Palabras clave :mecanización agraria, islas Baleares, agricultura tradicional, agricultura industrial, maquinaria agrícola.

\section{ABSTRACT}

A second exercise of reflection about the process of modernization of the Balearic agriculture is presented through the analysis of the mechanization. The work focuses on the Sixties decade and uses the Register of Tractors, specific source for the study of mechanization, non-exempt of problems but very useful for a detailed and diachronic analysis. In 1970, the end of the period, the mechanization has reached the whole territory, almost increasing by ten the power of agricultural machinery park in relation to that in 1950 .

Key words: agricultural mechanization, Balearic Islands, traditional agriculture, industrial agriculture, agricultural machinery.

Fecha de recepción: mayo 2013.

Fecha de aceptación: febrero 2014. 


\section{DEFINICIÓN DEL TEMA}

Se trata de un segundo ejercicio de reflexión sobre el proceso de modernización de la agricultura insular en los últimos cincuenta años a través del análisis de la mecanización, un aspecto representativo del tránsito de una agricultura tradicional, caracterizada por una escasa dependencia exterior, a una agricultura industrial, consumidora de inputs industriales y con un balance energético negativo. La mecanización es un indicador esencial para conocer la transformación de una agricultura tradicional u orgánica, fundamentada en el uso de energía animal y humana, hacia una agricultura moderna, que incorpora el uso de energía fósil (Naredo, 1988). En este caso, la aproximación al conocimiento del proceso de mecanización se ha centrado en la incorporación de maquinaria agrícola, en base a la extracción de datos del registro de tractores de las Islas Baleares, a partir de 1946 y hasta 1970.

De acuerdo con David Grigg (1992), la tierra y el trabajo son los principales inputs en la agricultura tradicional que ocupa más del $70 \%$ de la población activa y en la que las disponibilidades de energía se reducen a la animal y humana, el uso de materia orgánica es la principal fuente de fertilización, los rendimientos por activo agrícola y por hectárea son bajos, existe un elevado autoconsumo (de manera que se comercializa menos del $50 \%$ de la producción) y el grado de especialización de cultivos es escaso. Aceptando la definición de Grigg y las consideraciones iniciales, es posible afirmar que las Baleares se hallaban durante la postguerra en una situación de tránsito de la agricultura tradicional hacia una agricultura moderna. Sin duda, la Guerra Civil había abortado una progresión más rápida pues, de hecho, existe una continuidad en la secuenciación de la historia agraria española entre los cambios que tuvieron lugar desde finales del siglo XIX y lo ocurrido durante las décadas de los cincuenta y los sesenta. Así, para Clar (2008: 141):

«la reestructuración del sector agrario español, antes de la Guerra Civil y después de 1950 ha de considerarse una misma secuencia, con un período de freno, e inclusive de retroceso, entre ambos períodos. En esencia, los factores que propiciaron la transformación agraria en la España de los años cincuenta y sesenta se hallan ya después de la Primera Guerra Mundial...».

Ciertamente, el proceso de mecanización agraria con maquinaria autopropulsada iniciado en la década de 1940 es uno de los fenómenos más decisivos del cambio y la transformación de la agricultura insular que durante las décadas posteriores (de 1950 a 1980) haría esfuerzos para convertirse en una agricultura moderna, industrializada.

\section{OBJETIVOS}

Los estudios sobre la mecanización del campo en las Baleares son escasos y la información es, en general, muy dispersa. Nuestro objetivo es incidir en la evolución de la mecanización hasta la actualidad, particularmente en base al análisis de la progresiva incorporación de una maquinaria autopropulsada (formada primero por tractores a los que se añadirían motocultores, cosechadoras y tractores articulados, entre otros). Tras una primera aproximación al estudio de los inicios de la mecanización del campo entre los años 1946 y 1960 (Binimelis, 
Ginard, Ordinas, 2005), se plantea un segundo capítulo en el que se aborda la década de los sesenta, período central en el proceso de generalización en el uso de la maquinaria agrícola en Baleares, como en el resto de España. Naredo (1988: 19), refiriéndose a las décadas de los sesenta y setenta, apunta que «En lo que concierne al proceso de mecanización agraria se puede decir que lo característico de las dos últimas décadas ha sido su generalización entre las explotaciones de menor tamaño...».

Lógicamente, no es posible reducir la mecanización y la motorización del campo al uso de maquinaria autopropulsada sino que, paralelamente, también han de considerarse la electrificación de las explotaciones, la motorización de los pozos y de la extracción de agua, el avance en los complementos y herramientas para tractores (Clar, 2009) o la mecanización de las actividades ganaderas, entre otros muchos aspectos (López Ortiz, 1999: 86). No obstante, nos centraremos en el análisis de los vehículos matriculados.

Nuestro trabajo se plantea entre 1960 y 1970, período que abarca la etapa de desarrollo económico y urbano que se iniciaba con el Plan de Estabilización y que sucede al período de de la autarquía económica (1939-1959), a la vez que suponía la apertura a los mercados exteriores y la liberalización de los intercambios comerciales. No obstante, el cambio de signo para la agricultura española debemos situarlo ya a principios de la década de los cincuenta, coincidiendo con la toma de posesión de Cabestany como ministro de agricultura. Así lo señala Clar (2009: 105): «... puede decirse que 1951 significó para la agricultura lo que 1959 para el conjunto de la economía española, aunque sólo sea desde la perspectiva de una nueva orientación política que hubo de aguardar el impulso del período preestabilizador para hacerse realidad...». Por otra parte, es importante remarcar que 1960 representa en las Islas Baleares un punto de inflexión hacia la transformación económica y social impulsada por la especialización turística.

\section{SOBRE LA MECANIZACIÓN DEL CAMPO}

En España se constata la existencia de un número significativo de estudios sobre el proceso de mecanización de la agricultura, realizados mayoritariamente por historiadores económicos, economistas y geógrafos agraristas. La mayoría de ellos analizan sobre todo la modernización de la agricultura en el tránsito de la agricultura tradicional a la industrial, en las décadas posteriores a la Guerra Civil, siendo más escasos los relativos a etapas anteriores (Garrabou, 1990: 46).

Buena parte de estos trabajos son estudios de casos o monografías locales o regionales entre los que destacan estudios monográficos sobre la mecanización del campo en Andalucía (Ferrer, 1978) para el período de los años sesenta, y otro para las décadas de los 70 y 80 (Vico, 1996), Murcia (Andrés Sarasa, Espejo Marín, 1989) y Alicante (Segrelles, 1989) En el primer caso y basándose en los datos del Censo Agrario, Ferrer (1978) relaciona el proceso de mecanización en Andalucía con la estructura de la propiedad durante la década de los sesenta. Vico (1996), usando también los datos de los censos agrarios de 1972, 1982 y 1989, estudia las razones de la escasa mecanización del campo andaluz durante ese período, en relación al resto de España. El trabajo sobre Murcia es un análisis geográfico de la incidencia de la mecanización, relacionando su intensidad con los cambios en los usos agrícolas del suelo y, en una óptica productivista, con el aumento del PIB agrícola, de los rendimientos 
agrarios y del índice de mecanización. El de Alicante es un estudio de más empaque (se trata de una tesis de licenciatura editada en forma de libro) donde se analizan críticamente las diversas fuentes de información existentes que a su vez utilizará para relacionar dicho proceso con la estructura parcelaria y de la propiedad así como con los usos agrarios del suelo. De ámbito regional es también el análisis de Picazo y Reig (1990) sobre la mecanización y sustitución de factores productivos en la agricultura valenciana donde se pone de manifiesto la relación entre mecanización y expansión del regadío en un primer momento del proceso de difusión tecnológica. Por último, cabe recalcar el estudio de López Ortiz (1999) sobre la crisis de la agricultura tradicional y el tránsito hacia un modelo de agricultura industrial operado en Murcia, siendo la difusión tecnológica una variable más de las diferentes que se manejan para analizar ese tránsito.

Sin embargo, existen también algunos ejercicios de reflexión que intentan interpretar el proceso de mecanización en el marco del tránsito de una agricultura tradicional a una agricultura industrializada y que tienen todo el territorio español como ámbito de estudio. Mikel Buesa (1983) señala que la fabricación de tractores y de maquinaria agrícola se convirtió en uno de los objetivos fundamentales de la política industrial franquista, regulándose su fabricación a partir de un Real Decreto de 10 de febrero de 1940. No obstante, apunta que «habría que esperar al año 1953 para que, tras la declaración del sector de fabricación de tractores agrícolas como industria de interés nacional el año anterior, la política industrial alcanzara resultados efectivos en ese campo...» (Buesa, 1983: 226). De forma global, Clar (2008) interpreta que la Guerra Civil sólo supuso un revés coyuntural al proceso de cambio desde una agricultura orgánica a una agricultura industrial jugando, por supuesto, la mecanización un papel destacado en dicho proceso. El mismo Clar (2009) enfatiza el peso del factor institucional en el proceso de mecanización de la agricultura española durante la década de los 50. De carácter global es también la aportación de Naredo (2004) que vincula claramente el proceso de mecanización durante la década de los sesenta al desarrollo urbano e industrial y todo lo que ello comporta (salarios agrícolas, emigración a la ciudad, transición nutricional, etc.). Finalmente, cabe reseñar los trabajos específicos sobre la mecanización de Nicolás Ortega Cantero (1983) y el más reciente de Martínez Ruíz (2000), donde amplía hasta los años sesenta investigaciones anteriores que se detenían en 1932 (Martínez Ruiz, 1995). En relación a las Islas Baleares hay una ausencia notable de estudios específicos sobre dicha cuestión, a excepción de un primer ejercicio ya citado.

\section{III.1. Fuentes de información}

El estudio de la mecanización agraria en España es un tema «olvidado por los geógrafos» (Ferrer, 1978: 117) debido a las escasez de fuentes y «con frecuencia poco fidedignas y sin adecuada desagregación en la mayor parte de los casos» (Segrelles, 1988: 231). El estudio del proceso de mecanización de la agricultura cuenta con fuentes de información específicas y otras indirectas. En el primer grupo destacan los Registros de Maquinaria de las Jefaturas provinciales del Ministerio de Agricultura y el Censo de Maquinaria (CM), elaborados anualmente por las antiguas Cámaras Agrarias Locales. Entre las fuentes indirectas sobresale el Censo Agrario, que se elabora con una periodicidad de diez años desde 1962. Aunque en los censos de 1962 y 1972 ya aparece información provincial sobre maquinaria agraria, 
desglosada según el tamaño de las explotaciones, no será hasta el censo de 1982 cuando la información aparezca desagregada por municipios y aún así, en general, es calificada de forma crítica como insuficiente (Segrelles, 1988: 241). Se trata, por otro lado, de una fuente indirecta, además de sincrónica, que informa sobre el estado de la mecanización del campo en el momento concreto de elaboración del censo, por lo que no nos permite analizar el proceso de difusión de la innovación técnica.

El Censo de Maquinaria al Uso (denominado CM desde 1977 y 2-T desde su inicio en 1969 hasta 1977), elaborado por las Cámaras Agrarias locales a instancia del Ministerio de Agricultura con una periodicidad anual, ofrecía información detallada con un nivel de desagregación municipal de los distintos tipos de maquinaria agrícola, autopropulsada y arrastrada. Los secretarios de las cámaras locales eran quienes se encargaban de cumplimentar los cuestionarios limitándose, muchas veces, a copiar los datos del año anterior. Así lo pone de manifiesto Segrelles (1989: 20) en su estudio sobre la provincia de Alicante: «No podían estar corregidos de ninguna manera cuestionarios donde de forma manifiesta se habían copiado los distintos valores de un año para otro» que puede ser consecuencia de «la imposibilidad de controlar anualmente el parque de maquinaria y las nuevas adquisiciones...». Según el mismo autor, «no se puede estudiar una evolución anual estricta...», sino más bien los cambios producidos después de períodos más o menos prolongados. En definitiva, a pesar de la desagregación municipal, debemos dudar de la fiabilidad de esta fuente, tanto en el análisis temporal del proceso de difusión como en la contabilización real de las máquinas existentes.

Una primera premisa para llevar a cabo el estudio era la importancia de disponer de información original e inédita, objetivo que se ha conseguido en la elaboración de la base de datos y la posterior explotación de la serie de libros que forman el Registro de Tractores. Tal como ya explicó Segrelles (1989), su explotación es una tarea ardua y difícil a la que nunca ha dedicado empeño la Administración siendo, sin duda, la mejor documentación del proceso de difusión de la maquinaria autopropulsada con energía fósil en la transición de la agricultura tradicional a la agricultura industrial en el Estado español (Naredo, 1988).

Respecto a la fuente usada en la elaboración del trabajo, el Registro de Tractores del Ministerio de Agricultura, Jefatura Agronómica de Baleares, serie de la que se han consultado los primeros 7 volúmenes, además del volumen de Cosechadoras. Se trata de una serie específica para el análisis del proceso de difusión e implantación de la maquinaria agrícola en España que se inicia en 1946, teniendo en cuenta que hasta 1977 eran la delegaciones del Ministerio de Agricultura, "...las que otorgaban las matrículas de los tractores, motocultores o cosechadoras...» (Segrelles, 1988: 232). Dicha serie, como se podrá comprobar al especificar las características que recoge de cada máquina, cambia de formato a la vez que se producen pequeños cambios sobre las características recogidas de cada máquina inscrita. Permite conocer, desde 1946 hasta 1970 (hasta la inscripción 6356 en el caso de Baleares), no solamente el municipio de adscripción de la máquina, sino también el topónimo de la finca del adquirente, incrementando notablemente las potencialidades de la información al convertir prácticamente la explotación en la unidad de análisis usada. En nuestro caso, el grado de desagregación de los datos será el municipal, aunque no se descarta analizar el proceso de difusión de las innovaciones tecnológicas a nivel de cada una de las explotaciones, con la inestimable ayuda de la referencia toponímica de la empresa agraria. Por otra parte, se trata de una fuente diacrónica, pues permite seguir tanto la evolución en la inscripción de nueva maquinaria como también la de traspasos de máquinas de 
segunda mano procedente de otras provincias españolas. En un primer período (hasta el libro 7), no se distingue entre vehículos nuevos y usados, lo que da lugar a una ardua tarea al tener que diferenciar entre vehículos nuevos y traspasos a partir de la matrícula y al hecho de que normalmente en el apartado de observaciones se anota el número de inscripción de procedencia anterior así como el número de inscripción posterior. En este sentido estamos de acuerdo en que «...estos registros de inscripciones podrían depurarse para constituir una fuente idónea de estudio de la mecanización agraria, pero esto sería arduo y laborioso...» (Segrelles, 1988: 233). Tanto es así que ha sido en esta labor en la que nos hemos afanado y que ha supuesto la confección de la base de datos. Asimismo, al informar del número de caballos de vapor de cada una de las máquinas, se ha podido medir el peso de la maquinaria agrícola insular, algo que es imposible cuando simplemente se distingue entre tipos de máquinas como es el caso del Censo Agrario. Es sabido que en la construcción de tipologías de agricultura se acude al número de CV para medir el peso de la maquinaria agrícola de un país o de una región (véase al respecto el trabajo del geógrafo polaco Jerzy Kostrowicki (1991). Por otra parte, hasta 1977 -año en que tiene lugar la llamada «Operación Bloque», que consistió en otorgar a las Direcciones Generales de Tráfico la responsabilidad de matricular la maquinaria agrícola autopropulsada, previa inscripción en las delegaciones del Ministerio de Agricultura-, toda la maquinaria agrícola quedaba registrada en el Registro de Tractores. Es decir, nuestra base de datos, que finaliza en 1970, cuenta con cosechadoras y también motocultores o tractores articulados. A partir de 1977 se constituyó un libro de registro para cada tipo de maquinaria, razón por la cual se ha consultado el primer libro de registro de cosechadoras, que incluye también las inscritas en el anterior Registro de Tractores.

\section{EVOLUCIÓN, DIFUSIÓN TERRITORIAL Y CARACTERÍSTICAS DE LA MAQUINARIA AGRÍ- COLA EN LAS ISLAS BALEARES}

\section{IV.1. Evolución de la maquinaria agrícola}

El primer tractor de combustión interna aparecía en los Estados Unidos en 1897, fabricado por los norteamericanos Hart y Parr, aunque no fue presentado en público hasta 1907. En España, la oferta de tractores durante el primer tercio del siglo XX fue pobre, deficiente y de origen extranjero. En la década de 1920, el gran protagonista fue el modelo Fordson, fabricado por Ford, un tractor de 22-25 HP, muy adecuado para pequeños y medianos agricultores. En los años 1930 el número de tractores ligeros en España disminuía, puesto que «...la mayor parte de las máquinas importadas durante los años treinta fueron tractores de cadenas, en lugar de ruedas, y tractores de gran potencia, en lugar de los tradicionales Fordson...» (Martínez Ruíz, 2000: 133).

La Guerra Civil repercutió en la evolución de la agricultura española de las primeras décadas del siglo XX, pues supuso una clara ralentización del cambio técnico y de las disponibilidades energéticas. Durante la década de 1940 se evidencia nuevamente un predominio absoluto de la tracción animal, que representaba un 94,7\% del total de las disponibilidades energéticas. También se produjo un retroceso en la progresiva vinculación con el mercado y en especialización de los cultivos, con lo cual se mantenían las características de una agricultura orgánica o preindustrial. 
Tal como ya se apuntó en trabajos anteriores (Binimelis, Ginard, Ordinas, 2005) en las Islas Baleares, el registro de tractores y de maquinaria agrícola autopropulsada comienza en 1946. Se constata que durante aquel primer año se matriculó un elevado número de vehículos, sin duda a causa de la acumulación de registros que supondría la inclusión de la maquinaria que estaba operativa antes de 1946. Según el censo de maquinaria agrícola de 1932, había en las Baleares un total de 54 trilladoras y 54 tractores, siendo entonces una de las provincias más mecanizadas de España. Mientras que la media insular era de 5,6 HP/ha, la media estatal se situaba en 5,2 HP/ha (Martínez Ruíz, 2000). No obstante, el incremento del número de tractores matriculados se aceleraba después de 1954 hasta 1960, lo cual permitiría confirmar algunas hipótesis planteadas en estudios de carácter más general, como la que ya apuntaba Ortega Cantero (1983: 111): «...el primer episodio de intensificación del proceso de mecanización (aproximadamente 1954-55 a 1960) supone una generalización de la maquinaria que conlleva la introducción de unidades de pequeña potencia (debido tanto a disponibilidades de la oferta como a limitaciones de la demanda)». Aún así, el aumento generalizado de los tractores y de la maquinaria agrícola no se producirá hasta la década de los sesenta, pues si exceptuamos la maquinaria inscrita en 1961 (108 máquinas) que supone un ligero descenso respecto del último año de la década de los 50 (138 máquinas en 1960), a partir de ese momento las cifras siempre superan con creces los registros de la década de los cincuenta. En 1963 se alcanza la cifra de 642 máquinas registradas y aunque se produzca una substancial inflexión en los años 1964 (308 máquinas) y 1965 (330 máquinas), a partir de entonces el número se dispara con más de mil unidades anuales de nueva maquinaria en los años 1969 y 1970 (con 1.026 y 1.066 máquinas, respectivamente).

Figura 1

EVOLUCIÓN DE LAS MÁQUINAS INSCRITAS

EN EL REGISTRO DE TRACTORES POR TIPOLOGIIAS

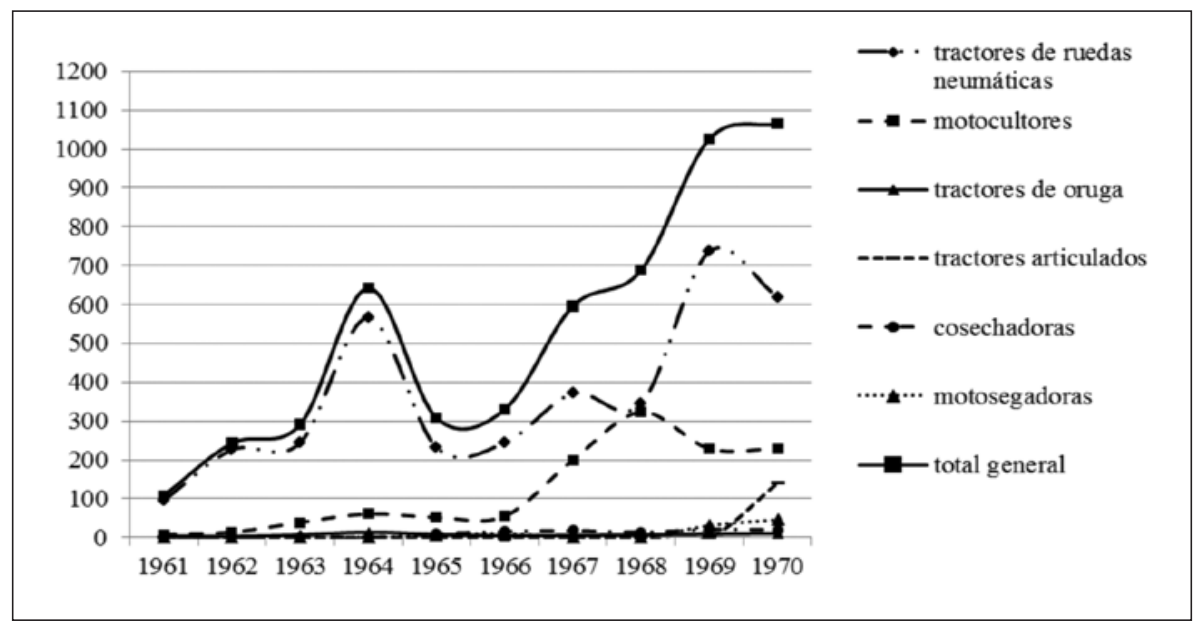

Fuente: Registro de Tractores del Ministerio de Agricultura. Jefatura Agronómica de Baleares (1946-1970). Elaboración propia. 
Por tipologías (Fig. 1), cabe destacar la introducción mayoritaria de tractores de ruedas neumáticas, (3.679 durante la década) con una tendencia ascendente sólo interrumpida por un ligero retroceso durante los años 1965 y 1966, para continuar al alza hasta el final de la década. Por otra parte, los motocultores se convierten en la segunda tipología en importancia, gracias al gran aumento experimentado en la segunda mitad de la década estudiada, sin lugar a dudas como consecuencia del ascenso experimentado por el cultivo en regadío de hortalizas. Paralelamente, resulta testimonial el peso de los tractores de oruga, relegados a explotaciones de montaña y de gran pendiente después de una mayor relevancia en la década de los cincuenta. En cambio, debemos reseñar la aparición, también a finales de los sesenta, de las cosechadoras (101) -que supondrán una revolución en el cultivo de cereales, donde aún la recolección precisaba de gran cantidad de mano de obra y de tracción animal, a pesar de la existencia de trilladoras mecánicas-, los tractores articulados (140 inscritos, todos en 1970) y las motosegadoras (94), ligadas al auge del cultivo en regadío de hortalizas y forrajes para el ganado. En definitiva, la mecanización agraria en la década de los sesenta revela una evolución en el uso de tecnología automotriz, protagonizada por la generalización del tractor de ruedas neumáticas en el conjunto de explotaciones agrarias de las Baleares, siendo esta implantación más intensa en la segunda parte de la década.

Además de la maquinaria nueva que se añade al parque existente, es considerable el dinamismo en el número de traspasos de máquinas entre explotaciones de la propia provincia o por compra de maquinaria de segunda mano que procede del resto de provincias españolas. De este modo, se produjeron un total de 827 traspasos de maquinaria agrícola entre 1961 y 1970, iniciado con 42 traspasos en 1961 y que alcanzaría cifras mucho más importantes en los años finales del período de estudio con 106, 137 y 181 traspasos en 1967, 1969 y 1970 , respectivamente, con una ligera inflexión en esa tendencia alcista de la segunda mitad de los sesenta que supusieron los 92 traspasos registrados en 1968. Por tipologías, resulta abrumadoramente mayoritario el traspaso de tractores de ruedas neumáticas (con 754 unidades), muy alejado del número de motocultores (47) y de los tractores de oruga (20). Testimoniales son los 4 traspasos de cosechadoras y los 2 traspasos de tractores articulados registrados ya al final de la década.

Por otra parte, un $16 \%$ de esos traspasos corresponde a máquinas llegadas de otras 25 provincias del Estado español (131 máquinas de un total de 827 traspasos), destacando las de Ciudad Real, Madrid, Alicante, Toledo y Valencia, con más de 10 traspasos, seguidas de Navarra, Barcelona y Lleida, con entre 5 y 10 traspasos. Por debajo se sitúan Badajoz, Sevilla, Valladolid, Albacete, Cáceres, Córdoba, Huelva, Logroño, Castellón y Cuenca, con menos de 5 traspasos y, finalmente, Girona, Guadalajara, Huesca, Jaén, Palencia y Teruel, con sólo 1 traspaso.

Durante la década de los sesenta se observa la expansión de la maquinaria agrícola de forma masiva gracias a la consolidación de marcas estatales de fabricación de maquinaria agrícola (Ebro, Barreiros) y a la penetración de firmas internacionales que establecen factoría propia en terreno hispano (Massey Ferguson, John Deere, Pascuali).

En la década de los 50, la menor difusión que tuvo la tecnología en el campo insular obedece a un proceso muy mediatizado (Clar, 2009:125), en el que la mecanización agraria en España se produce por una innovación inducida institucionalmente. Al existir más demanda que oferta, se impuso un sistema de reparto, con unos costes de gestión que ralentizaron el 
proceso de mecanización (Clar, 2009: 124) y que favorecieron a las grandes explotaciones en detrimento de los pequeños agricultores, de forma que la administración animó «a la salida del campo de los numerosos marginados del proceso» (Clar, 2009: 125). Así también ocurrió en Baleares, tal como pusimos de relieve en un estudio sobre el período 1947-1960, donde ya se constató como los grandes propietarios de grandes fincas fueron los primeros en solicitar y obtener un tractor (Binimelis, Ordinas, Ginard, 2005). No obstante, ya a finales de los sesenta, la generalización de la mecanización pone en alerta a la administración al considerar que la compra de tractores y afines da lugar a la inmovilización de un capital invertido en maquinaria que a lo mejor no se necesita sino temporalmente.

\section{IV.2. Implantación territorial}

En 1960 (Binimelis, Ginard, Ordinas, 2005: 121):, la maquinaria agrícola autopropulsada, en su mayoría tractores, está presente prácticamente en la totalidad de los municipios de Baleares. Las únicas excepciones son Estellencs, Banyalbufar, Deià y Escorca, todos ellos municipios mallorquines de montaña y con escasa población, así como el diminuto y urbano municipio de Eivissa. En 1965, la maquinaria agrícola autopropulsada está presente en todo el territorio de las islas si exceptuamos los municipios de Deià y Escorca.

En 1970, el peso de la maquinaria agrícola de Baleares se había multiplicado por 2,5 en relación al que tenía en 1965 (162.284 CV frente a 65.106 CV). Paralelamente, también aumentó la superficie labrada censada que, según el Censo Agrario de 1962, era de 246.699 ha, mientras que en 1972 se situaba ya en las 295.150 ha. A pesar de este aumento de las tierras cultivadas, la intensidad de la potencia mecánica autopropulsada casi se duplicó, ya que si en 1965 había de media 0,264 CV/ha, en 1970 dicha media se había situado en 0,549 $\mathrm{CV} / \mathrm{ha}$, tomando como referencia los datos de los censos agrarios de 1962 y de 1972. En esta fecha ya no sólo no existe ningún municipio que sea ajeno al proceso de difusión de dichas innovaciones técnicas, sino que además aumenta su intensidad en el resto del territorio insular.

Por otra parte, si se analiza la relación entre el número total de caballos-fuerza y la superficie cultivada (Fig. 2), la mayor intensidad del grado de mecanización corresponde a los municipios de Palma, sa Pobla y Muro, donde durante esas décadas se desarrolló una agricultura intensiva de regadío abocada a la producción de forrajes para la ganadería de leche, y de hortalizas y patatas para la exportación a la par que florecía una importante industria de transformación de productos lácteos y cooperativas de distribución cuyo objetivo fue alimentar el mercado exportador de patata temprana. En estos municipios y en esa década, la dependencia de la energía fósil no solamente se materializará por la aparición y difusión de los tractores, sino también por la sustitución de la energía eólica que movía los molinos de la huerta del Pla de Sant Jordi (Palma) por la energía fósil de los motores de explosión de las nuevas bombas de extracción de agua (Rosselló Verger, 2006). Todo ello convertiría estos municipios en el paradigma del éxito de un nuevo modelo de agricultura industrial integrada en el mercado y alejada del modelo tradicional.

En segundo lugar, se situarían los municipios de Alcudia (1,05 CV/ha), Pollença $(1,12$ $\mathrm{CV} / \mathrm{ha}$ ) y Montuïri (1,06 CV/ha), con casuísticas diferenciadas. Tanto Pollença, en la Serra de Tramuntana, como su vecina Alcudia, eran municipios litorales de gran desarrollo turístico y 


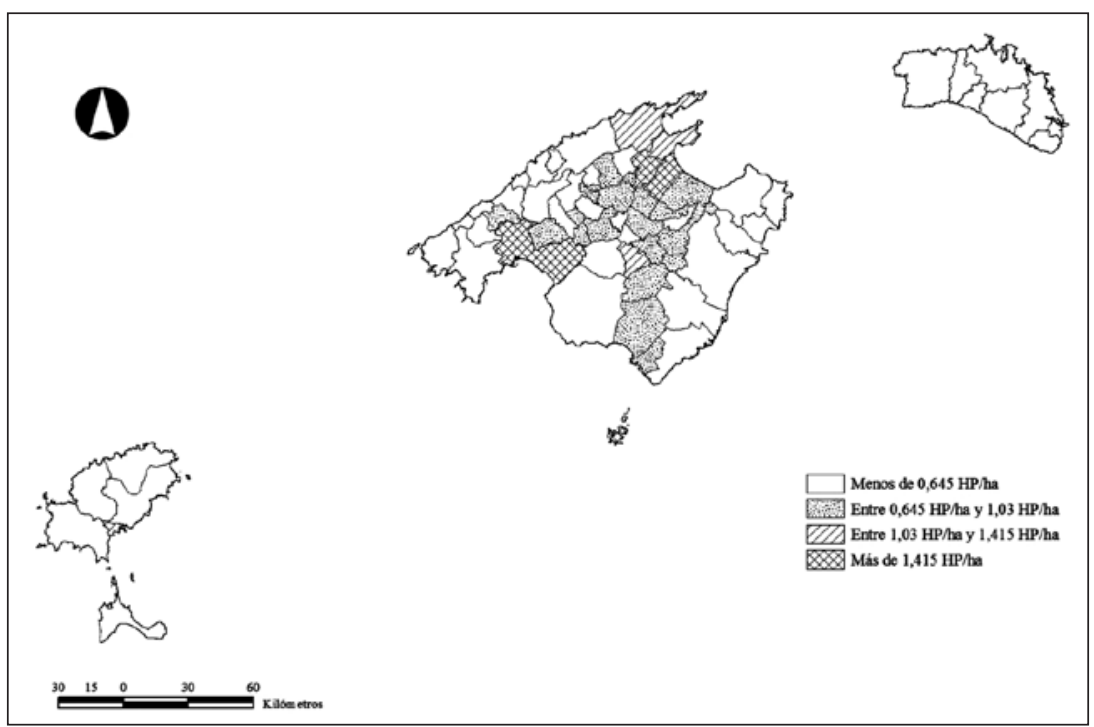

Fuente: Registro de Tractores del Ministerio de Agricultura. Jefatura Agronómica de Baleares (1946-1970). INE: Censo Agrario 1972. Elaboración propia.

con una superficie cultivada escasa. Aunque en ambos casos, con una parte, la colindante con sa Pobla, de regadío e intensiva. Por su parte, Montuïri, un municipio del Pla, donde en los sesenta destacaban los cultivos de la viña y del albaricoque junto a su dedicación cerealista. Igual que ocurría con la práctica totalidad de los municipios situados en el tercer nivel, que incluye los municipios de Santa Margalida, Petra, Villafranca, Sant Joan, Sineu, Maria de la Salut, Santa Eugènia, Sencelles y Porreres, en el Pla de Mallorca; además de Campos y ses Salines, en el Migjorn; y de Selva, Lloseta, Inca, Búger, Consell y Marratxí en el Raiguer, a los que hay que añadir el de Puigpunyent en la Serra de Tranuntana. El resto de municipios de la isla, básicamente en la Serra de Tramuntana, el Migjorn y el Llevant de la isla, presentan índices de mecanización situados en el último de los intervalos. Se trata de municipios que, por condicionantes orográficos o estructurales (Serra de Tramuntana, Serres de Llevant, Marina de Llucmajor) y de dimensión geográfica relativizan el avance que hubieran podido desarrollar en la difusión de la mecanización agrícola. Lo mismo ocurre en la totalidad de los municipios de Menorca y de las Pitiusas, a excepción del municipio de Eivissa, cuya extensión geográfica muy reducida favorece el alto grado de intensidad relativa alcanzado.

\section{IV.3. Urbanización funcional y modernización de la agricultura insular}

La relación entre la intensidad del grado de mecanización (CV/superficie cultivada) y el peso de la población activa agraria, con datos sólo disponibles para la isla de Mallorca de 1970 (Fig. 3), es poco significativa desde el punto de vista estadístico (correlación de 
Pearson de 0,2). Ello pone en alerta de que la transformación socioeconómica intensa que se inicia con el turismo y el reajuste de la agricultura en su tránsito a la modernidad, estando relacionados, son variables que no operan de forma lineal y en una única dirección, porque la realidad agraria isleña (aprovechamientos, estructura agraria y parcelaria) es compleja y dispar. Esa disparidad se puede observar agrupando los municipios en cuatro bloques (en función a su posición en relación a la media de las dos variables) que reflejan los cambios estructurales que los procesos de turistización-urbanización habían causado. En el primer grupo (municipios con una intensidad de mecanización superior a la media insular y un porcentaje de población activa agraria también superior a la media) se sitúan los municipios con gran especialización hortícola (casos de sa Pobla y Muro) o situados en la parte oriental de la comarca del Pla, de gran tradición cerealícola (Sencelles, Sant Joan, Petra, Maria de la Salut, Montuïri, Porreres, Vilafranca, Santa Margalida y Llubí), pero también con importantes áreas dedicadas a la viña, al albaricoque, al almendro e, incluso, a la alcaparra, todos ellos productos vinculados a la industria agroalimentaria de transformación.

Al segundo grupo (municipios con una intensidad de mecanización superior a la media insular y un porcentaje de población activa agraria inferior a la media) pertenecen Palma, capital insular con un alto grado de urbanización pero con un índice de mecanización muy alto; además de Inca, Lloseta y Consell, municipios del Raiguer, de tradición fabril; y Alcúdia y Pollença, municipios turísticos del extremo norte insular.

Figura 3

RELACIÓN ENTRE INTENSIDAD DE MECANIZACIÓN Y POBLACIÓN ACTIVA AGRARIA

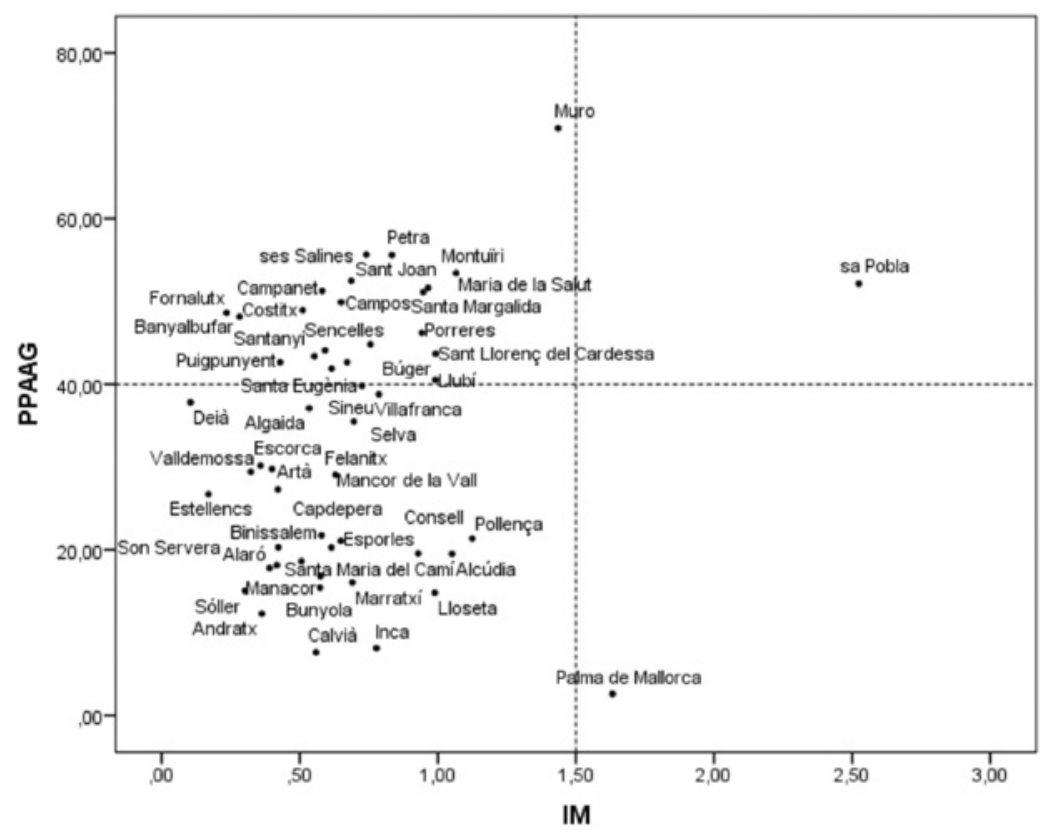

Fuente: Registro de Tractores del Ministerio de Agricultura. Jefatura Agronómica de Baleares (1946-1970). Quintana Peñuela, A. (1979). Elaboración propia. 
El tercer grupo (municipios con un porcentaje de población activa agraria superior a la media insular y con un índice de mecanización inferior a la media) reúne áreas geográficas dispares del centro y Oeste del Pla, del Raiguer, de la Serra de Tramuntana, y también de las Serres de Llevant y del Migjorn que, a excepción de Campos y ses Salines (ganadería intensiva de vacuno y forrajes de regadío), son de escasa superficie cultivada y de agricultura extensiva.

Por último, el último grupo (municipios con el porcentaje de población activa agraria y el índice de mecanización inferior a la media) está formado también por áreas de casuísticas dispares y que no responden a un único patrón, tanto de la Serra de Tramuntana como de su piedemonte (Raiguer), con poca superficie cultivada o de de tradición fabril, así como grandes municipios de las marinas del Llevant y del Migjorn con un alto grado de mecanización en cifras absolutas (número total de $\mathrm{CV}$ ), pero de gran extensión geográfica.

La desaparición de activos del campo vino acompañada de una creciente mecanización (véase Tab. 1), ajuste característico en la modernización de la agricultura española (Ortega Cantero, 1983). Ese ajuste se puede apreciar en otros contextos, como el murciano, donde «...todos los procesos de modernización técnica de la agricultura estuvieron guiados por dos objetivos: ahorrar mano de obra en los diferentes capítulos que formaban parte del proceso productivo y conseguir unos mayores rendimientos unitarios...» (López Ortiz, 1999: 90). En general, en los años 1950 comenzaba la mecanización del campo y la modernización de la agricultura. Como ha indicado Martínez Ruíz (2000: 17), «...el crecimiento industrial del país a partir de la década de 1950 y, mejor aún, a partir de los años sesenta, ejerció efectos profundos en el mercado de trabajo rural y en las disponibilidades de insumos modernos sin los cuales resulta difícil entender el fin de la agricultura tradicional en España». El proceso de mecanización y de adaptación tecnológica también ha sido, en opinión de Ortega Cantero (1983: 102), «...causalmente inseparable de la propia dinámica urbano-industrial».

El papel que en el conjunto de España ha podido representar el desarrollo industrial y urbano, en las Islas Baleares se ha producido a remolque de la actividad turística. El crecimiento del turismo es el detonante del crecimiento urbano y de la penetración de la cultura urbana en las áreas rurales, a la vez que creaba un mercado laboral atractivo para la población activa agraria, que ya desde 1950 abandonaba el campo de manera progresiva e imparable.

Tabla 1

POBLACIÓN ACTIVAAGRARIA Y POTENCIA DE TRACCIÓN MECÁNICA

EN LAS ISLAS BALEARES (1950-1960-1970)

\begin{tabular}{|c|c|c|c|c|}
\hline Ã̃NO & Población Activa & HP & $\begin{array}{c}\text { Población } \\
\text { Activa / HP }\end{array}$ & $\begin{array}{c}\text { HP / Población } \\
\text { Activa }\end{array}$ \\
\hline 1950 & 70.437 & $2.829,0$ & 24,89 & 0,04 \\
\hline 1960 & 63.474 & $16.123,0$ & 3,9 & 0,25 \\
\hline 1970 & 33.318 & $162.543,1$ & 0,2 & 4,87 \\
\hline
\end{tabular}

Fuente: Binimelis, Ginard, Ordinas (2005) y Quintana Peñuela (1979). Elaboración propia. 
Sin embargo, sólo se adecúan a esa lógica (índice de mecanización alto y decremento substancial de la población activa agraria) algunos municipios como Palma, además de Llucmajor, Manacor y Felanitx, de gran extensión geográfica con pesos absolutos muy altos en el desarrollo de la mecanización, aunque inmersos en un contexto de cambio funcional (turismo, construcción e industrias afines). Paralelamente, son los municipios agrarios del Pla de Mallorca así como las áreas con gran desarrollo del regadío, las que compaginan una apuesta clara por la modernización de su modelo agrícola y la dedicación a la agricultura de buena parte de su población activa. Son espacios de agricultura intensiva, de secano o de regadío, y alejados todavía de la transformación funcional que impuso el proceso de turistización-urbanización insular. Por otra parte, el ajuste del modelo de producción agrícola a la transformación funcional tiene otras respuestas que indudablemente deben valorarse. De acuerdo con el Censo Agrario de 1972, el 65\% de los titulares de explotación ejercían una actividad principal no agraria y sólo el 34\% restante lo eran a tiempo completo. Es decir, la agricultura a tiempo parcial fue, ya desde el inicio, una respuesta del campo insular al cambio socioeconómico, aspecto también muy importante para comprender la dinámica de las explotaciones agrarias.

\section{IV.4. Características del parque de maquinaria agrícola}

\section{a) La potencia de la maquinaria agrícola usada}

La potencia de los tractores, en general, aumentó entre 1960 y 1970, aunque se produjo una cierta polarización (Fig. 4). En 1960, en relación a 1955, se duplicaron los vehículos de entre 21 y $30 \mathrm{HP}$, con 222 unidades que suponen el 29,9\% del total. La potencia inferior a $20 \mathrm{HP}$, a pesar de que también casi se dobló (con 137 unidades, de las cuales 18 eran motocultores), perdió peso relativo (24,6\% del total); mientras que los vehículos de entre 31 y $40 \mathrm{HP}$ se cuadriplicaron en cifras absolutas, a la vez que se duplicó su peso relativo, que pasó de un $12,2 \%$ a un $24,6 \%$. Al mismo tiempo, se incrementaron las unidades de 41 a $60 \mathrm{HP}$, con un $8,8 \%$. Asimismo aparecen en el registro 10 unidades (todas de tracción oruga) de más de $61 \mathrm{HP}$, aunque la potencia máxima se halla representada por dos máquinas de 130 HP cada una.

En 1965, la maquinaria con menos de 20 HP más que duplicó su número (20,6\% del total, en la que destacan motocultores y algunos tractores articulados), mientras que las máquinas con un intervalo de potencia de 21 a $30 \mathrm{HP}$ disminuyeron su peso absoluto a menos de la mitad y el relativo a una séptima parte (con 109 máquinas y sólo un 6,2\% del total). Paralelamente, las máquinas de entre 31 y $40 \mathrm{HP}$ se septuplican (720 unidades) pasando a representar un $40,6 \%$ del total. Por su parte, las máquinas de entre 61 y 80 HP se multiplican por 10 (500 unidades y un $28,3 \%$ del total). Así pues, las máquinas de entre 31 y 60 HP representaban casi un $70 \%$ del parque de maquinaria agrícola a mediados de la década de los sesenta. Por otra parte, las unidades de más de $61 \mathrm{HP}$ triplicaron su número (30 unidades), aunque su peso relativo continuó siendo escaso $(2,2 \%)$.

En 1970, el número de máquinas se triplica (con 5.277 unidades frente a 1.765 en 1965), afectando dicho aumento a todas las categorías. Las máquinas de menos de $20 \mathrm{HP}$ son las que experimentan un mayor aumento, casi quintuplicándose (1.989 unidades que 
representan un $37,7 \%$ ) como consecuencia del incremento de los tractores articulados en esta segunda parte de la década. Las máquinas con más de 21 HP y menos de 40 HP casi se triplican (342 unidades), aunque manteniendo un peso relativo similar al de 1965 $(6,7 \%)$. Menor fue el desarrollo de las máquinas de entre 31 y $40 \mathrm{HP}$, hegemónicas por su peso relativo en 1965 (1.295 unidades y un peso del 24,5\%). Y significativo ha sido el crecimiento absoluto de las máquinas de más de $61 \mathrm{HP}$, fruto también de la aparición de las cosechadoras, que sextuplican el peso que tuvieron en 1965 (con 182 unidades que equivalen a un $3,5 \%$ del total).

DISTRIBUCIÓN DE MAQUINARIA POR INTERVALOS DE POTENCIA (HP)

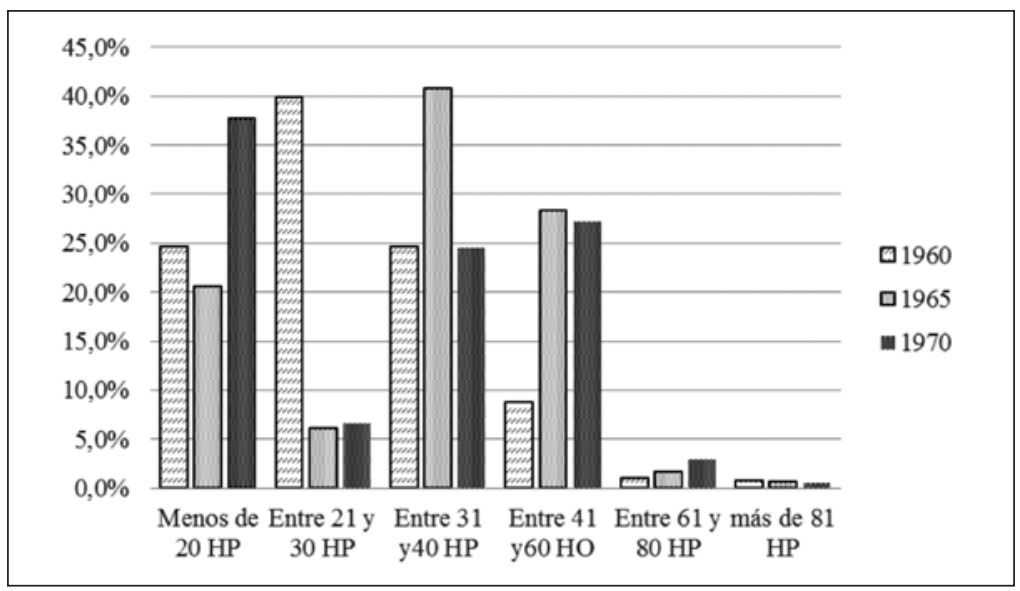

Fuente: Registro de Tractores del Ministerio de Agricultura. Jefatura Agronómica de Baleares (1946-1970). Elaboración propia.

\section{b) Tipo de combustible}

Los diferentes tipos de combustible usados evolucionaron desde el inicio del proceso de mecanización. La gasolina fue absolutamente predominante a principios de los años 50, pero hacia el final de la década ya se impuso el gasoil manteniéndose hasta el final del período estudiado. En cambio, el uso del petróleo y del fuel fue absolutamente testimonial. A finales de 1970 había en las islas 4.657 unidades que funcionaban con gasoil agrícola, 376 con gasolina (grupo en el que destaca la creciente importancia de los motocultores), 240 con petróleo, y sólo uno con fuel. Es evidente que, con el transcurrir de los años, el gasoil se fue imponiendo, a pesar del uso mayoritario de la gasolina en los motocultores de escasa potencia. El paso de un tipo de combustible a otro responde a un conjunto de factores estructurales, vinculados al cambio de la situación económica española. Como han indicado Abad y Naredo (1997: 254), «...al iniciarse la década de 1950, concurren otra serie de elementos ajenos al comportamiento del sector agrario, que actuaron a favor del desarrollo industrial, como pueden ser, por ejemplo, la ayuda americana y la desaparición de los estrangulamientos en el abastecimiento de energía». 
Por otra parte, es en la década de los sesenta cuando explota el uso de combustibles fósiles en el agro isleño, sustituyendo claramente el gasoil al petróleo tal como demuestran las cifras oficiales de carburantes bonificados (Fig. 5). Entre 1957 y 1968, el consumo de gasoil en la agricultura pasó de 3,5 millones de litros a un total superior a los 21 millones, mientras decae el uso de petróleo desde 1,63 millones de litros en 1957 a poco más de 0,5 millones en 1968. Ambos carburantes son usados tanto en maquinaria automotriz como en motores de explosión que mueven trilladoras o bombas de extracción de agua en las áreas de regadío. Resulta muy significativo el aumento del consumo de gasoil que se multiplica por 5,5 en una década, constituyendo la prueba irrefutable del cambio que se produce en el metabolismo agrario isleño, al sustituir el trabajo humano y la tracción animal por energía fósil, creadora de «dependencia extraterritorial» (Naredo, 2004: 498).

Figura 5

EVOLUCIÓN EN EL CONSUMO DE CARBURANTES BONIFICADOS

(1957-1968)

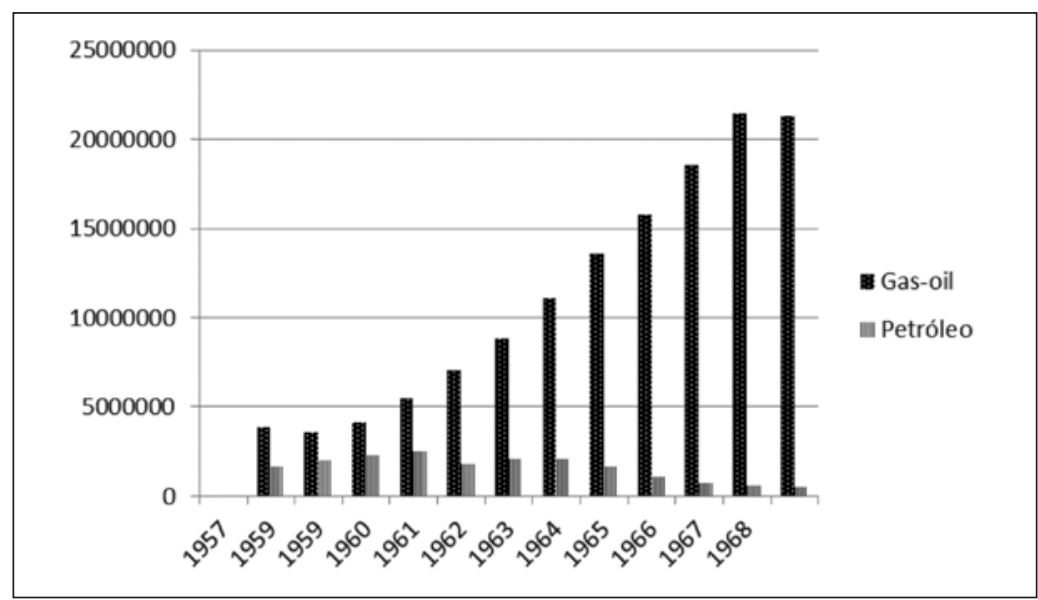

Fuente: Ponencias y Conclusiones del III Pleno del Consejo Económico Sindical Provincial de Baleares, Consejo Económico Sindical Provincial, 1965. Elaboración propia.

\section{IV.5. Coexistencia con las prácticas tradicionales}

Es evidente que la introducción de maquinaria agrícola se producía simultáneamente al mantenimiento de las prácticas de carácter más tradicional, por lo que se trata de un proceso de mecanización progresivo que sustituye y coexiste con el uso de la fuerza animal. De hecho, la ganadería equina jugó un papel preponderante en la organización de la explotación campesina que se fue consolidando tras múltiples episodios de fragmentación de la propiedad en las primeras décadas del siglo pasado. Aportaba la energía necesaria para el laboreo y cultivo de la tierra, de ahí que a mediados de los años cuarenta del siglo XX se apuntara la vital importancia que mantenía a pesar de su sustitución paulatina como medio de transporte: «En líneas generales el censo equino no ha sufrido en estos últimos veinte años variación apreciable. No sorprende esta aparente inmunidad a los factores de carácter accidental que 
tan funestos han sido para el resto de las especies que integran la ganadería. No se olvide que ya desde 1925, tanto el caballo como el mulo y el asno, al ser desplazados por el motor mecánico del campo de los transportes, se refugiaron en el reducto campesino, en donde su papel es práctica y teóricamente insustituible». (Anteproyecto de ordenación económicosocial de Baleares, 1947-1951).

A pesar de la importancia que a mediados de siglo pasado se seguía concediendo a la ganadería equina como fuerza de tracción en la actividad agraria, las cifras muestran como la decadencia es el signo que presidía el perfil de la curva que traza su evolución desde allá, en los años veinte, hasta principios de los sesenta, tal vez con la excepción de un leve repunte en los duros años de la posguerra. Así, el total de reses desciende desde 47.369 en 1925 hasta las 24.343 de 1966: (Binimelis, Ordinas, 2008). Dentro del conjunto equino, destaca la importancia numérica del ganado mular que llega a suponer hasta un $59 \%$ en 1965 , porcentaje que se debe explicar en la lógica de la importancia de la tracción animal en las explotaciones agrarias insulares.

La decadencia de la ganadería equina en general, y de la mular en particular, está directamente relacionada con la progresiva motorización del campo isleño, que se inició de forma tímida en las décadas de autarquía (Binimelis, Ordinas, Ginard; 2005) y se consolida en los años sesenta, inaugurando, junto con otros indicadores como el aumento de la ganadería intensiva de vacuno para la producción de carne y leche, la Revolución Verde en el contexto del campo isleño:

«Indudablemente, este tipo de ganado como sucede en todas las partes del mundo, decrece sensiblemente, dando paso a la fuerza motorizada, utilizando los terrenos que anteriormente se dedicaban a producir alimentos para este tipo de ganado, a seguir produciendo alimentos, pero ya para el ganado de carne o de leche». (Perspectivas de desarrollo económico de la provincia de Baleares, 1962: 64-65).

No obstante, continuó teniendo peso específico en las labores del campo durante los primeros años de cambio agrario, allá por los años sesenta:

«El trabajo que el ganado de labor ha realizado desde siempre, va poco a poco siendo sustituido por la máquina, en beneficio, sin duda, de los rendimientos trabajo-producción del campo, pero con ciertos inconvenientes que sería necesario subsanar o prevenir. Sin embargo, el grado de mecanización no es tan elevado como para presentar un serio peligro; es más, hoy las islas están insuficientemente mecanizadas... La causa de la deficiente mecanización es sin duda la deficiente dimensión de las explotaciones, que obliga a usar, para realizar las labores, de un elevado número de cabezas de ganado». (Ponencias y Conclusiones del III Pleno del Consejo Económico Sindical Provincial de Baleares, 1965).

A pesar del estallido de la tracción mecánica, persistían los sistemas de tracción animal, de la misma forma que se ha detectado en Murcia (López Ortiz, 1999: 86). No obstante, en el período inicial estudiado, anterior a 1960, el arado con el uso de la fuerza animal era 
mayoritario e incluso se documenta un caso en que el tractor se usaba para mover arados que habían sido fabricados para tracción animal (Col·lectiu Teranyines, 2003: 98). Arar y cultivar son, tal vez, las labores del campo que requieren más inversión de energía y de trabajo, de ahí que fuesen las actividades más afectadas por la mecanización. La preeminencia del arado y de los cultivadores obedece al hecho que tradicionalmente son los útiles más importantes en el ciclo agrícola.

\section{CAMBIOS DERIVADOS DE LA MECANIZACIÓN}

La progresiva mecanización del campo en la década de los sesenta incidió en la reducción de la inversión en trabajo durante todo el ciclo agrario, repercutiendo en primer lugar en las labores de arar (uso del arado, de gradas, de discos, etc.) y de preparación del terreno antes de la siembra (uso de cultivadores, así como en las araduras superficiales en los campos dedicados a los frutales, de secano o de regadío, y a la viña gracias a la incorporación inicial de cavadoras). Además del tractor convencional, se introdujeron durante esta década los tractores articulados, de escasa cilindrada; los motocultores, de forma generalizada; y las motosegadoras; poniendo de manifiesto el auge experimentado por los cultivos de regadío, de forma que mecanización y aumento del regadío son los ejes en las islas, como también en el resto de España, de la modernización de las estructuras agrarias. Por otra parte, la consolidación de la trilladora, además de la introducción incipiente, ya a finales de la década, de las cosechadoras automotrices, contribuyó a la reducción del trabajo de recolección, la última fase del ciclo agrícola tradicional. Además, la mecanización también contribuía a la especialización de los cultivos y al abandono de las labores difíciles de mecanizar (siega bajo los árboles, compaginación de arboricultura y cultivo de cereales). Asimismo daba lugar a la transformación del sistema de cultivos, que se hacían más intensivos, o a la progresiva desaparición de los barbechos.

La intensificación de los regadíos fue también en Baleares uno de los ejes de la política agraria durante el franquismo. A finales de la década de los cuarenta ya se apostaba por la sustitución de los artilugios tradicionales para la elevación de agua propios de la economía natural (1900 molinos y 2.650 norias), por motores de gasolina (1.500) o eléctricos (550), en aras a aumentar la productividad agrícola porque «...norias y molinos (...) en muchos casos tienen limitado el caudal a una cantidad que no basta a cubrir las necesidades de riego más intensivo...», además de buscar «una orientación forrajera de sus cultivos que, menos exigentes en agua, ampliarían al máximo la zona regable y contribuiría a mantener la estabilidad de la ganadería...» (Anteproyecto de ordenación económico-social de Baleares, 1947: 248). Según el citado anteproyecto, había en las islas 12.000 has. de regadío. A finales de la década de los cincuenta «...en la provincia existe una superficie regable de 10.763 has. destinadas a cultivos herbáceos, 1.411 a frutales y 288 has. de arrozal...» (Anteproyecto de ordenación económico-social de Baleares, 1947: 77). Además se sugiere la sustitución parcial de la patata temprana por la patata tardía, los frutales (cítricos y otros) y las superficies de regadío «...que no se puedan destinar a los cultivos anteriormente citados, deben dedicarse a plantas forrajeras» (Anteproyecto de ordenación económicosocial de Baleares, 1947: 88) (fundamentalmente alfalfa). Se apuesta por la intensificación de los regadíos, con un cambio tecnológico que permita facilitar la elevación del agua 
subterránea, incentivando también la electrificación de los mismos. En 1964 había 14.974 has. de tierras regadas, aunque en la misma publicación se nos apunta que en 1959 eran 15.399 (Ponencias y conclusiones del III Pleno del Consejo Económico Sindical Provincial de Baleares, 1965), alcanzándose hasta 17.800 has. en 1968 y 18.952 has. en 1974 (Salvà, 1992: 719). La modernización de la agricultura de las islas significó un cambio en la orientación productiva de sus cultivos, apostándose por la especialización ganadera y las hortalizas (patata temprana para la exportación), y en definitiva por el regadío orientado a productos de mercado. Así se decía, ya en los años setenta, sobre lo ocurrido: «La estructura espacial de los cultivos ha presentado un incremento muy importante de la producción forrajera en detrimento de la producción cerealícola y de las leguminosas... notándose un desplazamiento hacia aquellos procesos de producción que pueden ser mecanizados fácilmente o encuentran una salida garantizada en el mercado...» (La agricultura de las Baleares, 1981: 98). En definitiva, la reorientación productiva y la apuesta por el regadío y el proceso de mecanización van de la mano y son dos de las variables que definen el tránsito de la agricultura tradicional insular.

Paradójicamente, por otra parte, el proceso de mecanización no trajo aparejado un aumento homogéneo del tamaño de las explotaciones agrarias, fruto de una concentración de las mismas. Las explotaciones agrarias de Baleares aumentaron de 41.856 en 1962 a 48.575 en 1972 (un 16\%). No obstante, este aumento no fue uniforme ni se dio por igual según tamaños ni tampoco según áreas geográficas. Durante la década de los sesenta las explotaciones menores de 5 has. aumentaron un 32\% (9.001 explotaciones en total). Esta profundización en el microfundismo se debe en primer lugar, a la práctica generalizada de la agricultura a tiempo parcial que «...permite completar la renta de la unidad familiar con ingresos procedentes de otras actividades...» (La agricultura de las Baleares, 1981: 58) y a la incipiente presión urbana sobre el campo, que paulatinamente permitió que la tierra dejase de ser factor de producción, para convertirse en riqueza. También aumentan los intervalos de explotaciones de 50 a 70 has. (18\%) y las de 200 a 300 has. (21\%), respondiendo la dinámica de estos dos intervalos al ajuste que ya la modernización de la agricultura representaba (concentración, mecanización, especialización).

La relación del índice de mecanización (IM: CV/has. cultivadas) con la dinámica de las explotaciones (DE: explotaciones en 1972/explotaciones 1962) es también poco significativa estadísticamente (correlación de Pearson de 0,4). No obstante, la distribución de puntos nos permite observar (Fig. 6) como el proceso de concentración es propio sobre todo de los municipios de Mallorca donde crece el regadío (Palma, Muro, sa Pobla) y aumenta la mecanización (cuadrante inferior derecha), y en los de Menorca que, aunque con discretos índices de mecanización, apostaron por la especialización ganadera (mecanización inferior a la media, cuadrante inferior izquierda). En segundo lugar, hay también áreas dispares donde aumentó la mecanización y el número de explotaciones como consecuencia del mantenimiento del microfundismo en un contexto de auge de la agricultura a tiempo parcial. Por último, (cuadrante superior izquierda), gran parte de los municipios de la Serra de Tramuntana, del Migjorn y Serres de Llevant en Mallorca y buena parte de los de las Pitiusas, se hallan escasamente mecanizados y con una estructura de explotaciones dual. 


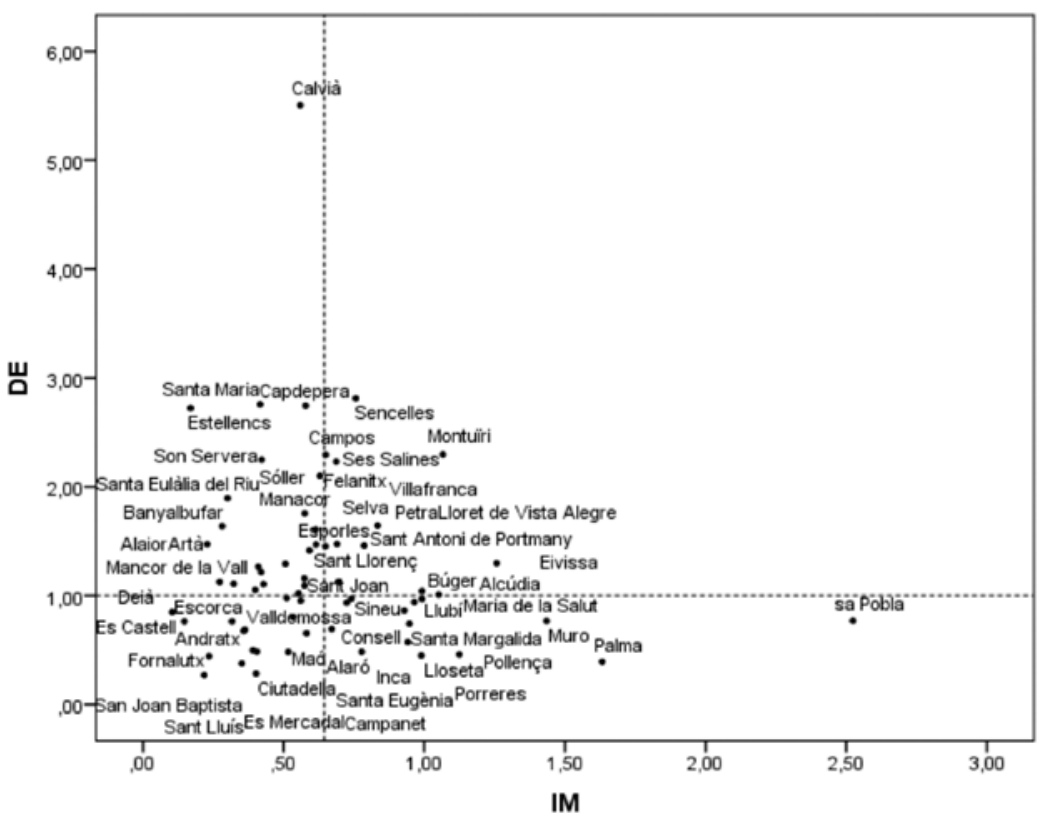

Fuente: Registro de Tractores del Ministerio de Agricultura. Jefatura Agronómica de Baleares (1946-1970). Censo Agrario 1972. Elaboración propia.

\section{CONCLUSIONES}

En el análisis del tránsito de la agricultura tradicional, de base orgánica, a la agricultura industrializada, el proceso de motorización y mecanización de las actividades agrícolas constituye un eje básico. El trabajo es un segundo ejercicio en el estudio del desarrollo de mecanización agraria de las islas Baleares, en su década determinante, los años sesenta. Se ha realizado a partir del Registro de Tractores, fuente específica y única para estudiar de forma diacrónica dicha evolución y llegar a las conclusiones que siguen.

1. En la década de 1950 comenzaba en las Baleares un tímido proceso de mecanización. Paralelamente, tanto a lo largo de esta década de forma incipiente como de forma intensa en la década de 1960, se producía un primer momento de desarrollo del turismo de masas, que ocasionaba un considerable éxodo rural interno y un no menos destacable éxodo profesional. La incorporación de los tractores a las labores agrícolas en 1960, aunque notable, todavía no alcanzaba la totalidad del territorio. De hecho, esta fase no se consolidaría hasta una década después, en 1970, cuando la motorización no sólo alcanzó la totalidad del territorio sino que además se multiplicó casi por 10 la potencia del parque de maquinaria agrícola respecto a la existente en 1950. Por otra parte, la intensidad de la potencia mecánica autopropulsada casi se duplicó, ya 
que si en 1965 había de media 0,264 CV/ha, en 1970 ésta se había situado en 0,549 CV/ha, tomando como referencia los datos de los censos agrarios de 1962 y de 1972.

2. La mecanización agraria en la década de los sesenta está protagonizada por la generalización del tractor de ruedas neumáticas en el conjunto de explotaciones agrarias de las islas, siendo esta implantación más intensa en la segunda parte de la década. La incorporación de nuevas tipologías de maquinaria en la segunda mitad de la década, como es el caso de los tractores articulados, los motocultores, las motosegadoras y también, de forma incipiente, las cosechadoras, contribuyeron a la intensidad del cambio tecnológico. De ahí que en 1970 el peso de la maquinaria agrícola de Baleares se había multiplicado por 2,5 en relación a la que tenía en 1965 (162.284 CV en 1970 frente a 65.106 CV en 1965), sólo 5 años antes.

Son los municipios de grandes dimensiones y, por tanto, con mayor superficie agraria cultivada (Llucmajor, Manacor y Felanitx), los que ocupan la hegemonía en la medida de la intensidad del proceso de mecanización en cifras absolutas Sólo destacar el mayor protagonismo adquirido por sa Pobla, municipio de dimensiones medianas en el conjunto insular, pero protagonista del despegue de la agricultura intensiva de regadío. De ahí el lugar hegemónico que ocupa ya al finalizar la década de los sesenta. Si se analizan datos relativos (CV/ha cultivadas), la mayor intensidad del grado de mecanización corresponde a los municipios de Palma, sa Pobla y Muro, convirtiéndose en el paradigma del éxito de un nuevo modelo de agricultura industrial integrada en el mercado. Por otra parte, son los municipios agrarios de Pla de Mallorca así como las áreas con gran desarrollo del regadío, las que compaginan una apuesta clara por el nuevo modelo de agricultura industrial y la vocación agraria de buena parte de su población activa.

3. Por otra parte, se observa la expansión masiva de la maquinaria agrícola en el campo gracias a la consolidación de marcas estatales encargadas de su fabricación (Ebro, Barreiros) y a la penetración de firmas internacionales que establecen factoría propia en terreno hispano (Massey Ferguson, John Deere). Destaca asimismo la creciente importancia adquirida por los tractores articulados de escasa potencia (Pascuali, BJR), en una década de crecimiento del regadío en las huertas del Prat de Sant Jordi y en los municipios de Campos, sa Pobla y Muro.

4. Se amplía el abanico de actividades del campo que han sido mecanizadas (mayor variedad de herramientas complementarias), además de la tipología de las máquinas inscritas (sobretodo tractores, pero también tractores articulados, motocultores, motosegadoras y la introducción incipiente de las cosechadoras), lo que pone de manifiesto el auge experimentado por los cultivos de regadío, de tal forma que mecanización y aumento del regadío, serán los ejes de la modernización de las estructuras agrarias tanto en las islas como en el resto de España.

\section{BIBLIOGRAFÍA}

ABAD, C. y NAREDO, J.M. (1997): «Sobre la «modernización» de la agricultura española (1940-1995): de la agricultura tradicional hacia la capitalización agraria y la dependencia asistencial», en Gómez Benito, C. y González Rodríguez, J. J. (eds.), Agricultura y socie- 
dad en la España contemporánea, Madrid, Ministerio de Agricultura, Pesca y Alimentación y Centro de Investigaciones Sociológicas, 249-316.

ANDRÉS SARASA, J.L. y ESPEJO MARÍN, C. (1989): «La mecanización de la agricultura en la región de Murcia». Revista de Estudios Agro-Sociales, n 147, 155-170.

Anteproyecto de ordenación económico-social de Baleares 1947-1951 (1947). Junta Provincial de Ordenación Económico-Social de Baleares.

BINIMELIS SEBASTIÁN, J., GINARD BUJOSA, A. y ORDINAS GARAU, A. (2005): «La mecanización agraria en las islas Baleares durante el período de la autarquía (19461960)». Investigaciones Geográficas, nº 38, 13-134.

BINIMELIS, J. y ORDINAS, A. (2008): «El tránsito del productivismo al postproductivismo en los sistemas ganaderos. El auge del ganado equino ligado a actividades de ocio en la isla de Mallorca», en Los espacios rurales españoles en el nuevo siglo. Actas XIV Coloquio de Geografía Rural, Universidad de Murcia. Asociación de Geógrafos Españoles, 15-22

BUESA, M. (1983): «Industrialización y agricultura: una nota sobre la construcción de maquinaria agrícola y la producción de fertilizantes en la política industrial española (1939-1963)». Agricultura y Sociedad, n 28, 223-249.

CLAR, E. (2008): «Más allá de 1936: la crisis de la agricultura tradicional española en perspectiva, 1900-1975». Ager. Revista de Estudios sobre Despoblación y Desarrollo Rural, $\mathrm{n}^{\mathrm{o}} 7,109-147$.

CLAR, E. (2009): «Contra la virtud de pedir... Barreras administrativas a la difusión de tractores en España: 1950-1960». Investigaciones de Historia Económica, nº 13, 97-132.

FERRER RODRÍGUEZ, A. (1978): «La mecanización del campo en Andalucía». Cuadernos de Geografía de la Universidad de Granada, $\mathrm{n}^{\circ} 8,117-129$.

GALLEGO MARTÍNEZ, D. (2001): «Sociedad, naturaleza y mercado: un análisis regional de los condicionantes de la producción agraria española (1800-1936)». Historia Agraria, $\mathrm{n}^{\mathrm{o}} 24,11-57$.

GARRABOU, R. (1990): «Sobre el atraso de la mecanización agraria en España (18501933)». Agricultura y Sociedad, $\mathrm{n}^{\circ}$ 57, 41-77.

GRIGG, D. (1992): The transformation of agriculture in the West. Oxford. Blackewll.

KOSTROWICKI, J. y SZYRMER, J (1991): Agricultural tyipology guidelines. Warszawa. Polish Academy of Sciences. Institute of Geography and Spatial Organization.

La agricultura de las Baleares (1981). Consell General Interinsular. Conselleria d'Economia i Hisenda. Banca March.

LÓPEZ ORTIZ, M.I. (1999): «Entre la tradición y el cambio: la respuesta de la Región de Murcia a la crisis de la agricultura tradicional». Historia Agraria, $\mathrm{n}^{\circ}$ 19, 75-113

MARTÍNEZ RUÍZ, J. I. (1995): «La mecanización de la agricultura española: de la dependencia exterior a la producción nacional de maquinaria (1862-1932)». Revista de Historia Industrial, $\mathrm{n}^{\circ} 8,43-63$.

MARTÍNEZ RUÍZ, J. I. (2000): Trilladoras y tractores. Energía, tecnología e industria en la mecanización de la agricultura española (1862-1967). Sevilla. Universidad de Sevilla - Edicions de la Universitat de Barcelona.

NAREDO, J.M. (1988): «Diez años de agricultura española». Agricultura y Sociedad, $\mathrm{n}^{\circ}$ 46, 9-36. 
NAREDO, J.M. (2004): La evolución de la agricultura en España (1940-2000). Granada. Universidad de Granada.

ORTEGA CANTERO, N. (1983): «El proceso de mecanización y adaptación tecnológica del espacio agrario español». Agricultura y sociedad, nº 27, 81-149.

Perspectivas de desarrollo económico de la provincia de Baleares (1962). Consejo Económico Sindical Provincial, marzo 1962, $\mathrm{n}^{\circ}$ 6A.

PICAZO TADEO, A. y REIG MARTÍNEZ, E. (1990): «Mecanización y sustitución de factores productivos en la agricultura valenciana». Agricultura y Sociedad, n 57, 9-40.

Ponencias y conclusiones del III pleno del consejo económico sindical provincial de Baleares (1959). Consejo Económico Sindical Provincial.

Ponencias y conclusiones del III pleno del consejo económico sindical provincial de Baleares (1965). Consejo Económico Sindical Provincial.

QUINTANA PEÑUELA, A. (1979): El sistema urbano de Mallorca. Palma. Editorial Moll.

ROSSELLÓ VERGER, V.M. (2006): Les illes, redescobertes: Mallorca, Menorca, Eivissa i Formentera. Barcelona. Publicacions de l'Abadia de Monserrat.

SALVÀ TOMÀS, P. (1992): «Las actividades agrarias en las islas Baleares en la etapa del turismo de masas», en El medio rural español. Cultura, paisaje y naturaleza: homenaje a don Angel Cabo Alonso, Ediciones Universidad de Salamanca, 715-730.

SEGRELLES SERRANO, J.A. (1988): «Fuentes para el estudio de la mecanización agraria». Revista de Estudios Agrosociales, $\mathrm{n}^{\circ}$ 146, 231-242.

SEGRELLES SERRANO, J.A. (1989): La mecanización agraria en la provincia de Alicante. Generalitat Valenciana, Conselleria d'Agricultura i Pesca.

VICO RUIZ, A. (1996): «La mecanización del campo en Andalucía según los censos agrarios de 1972, 1982 y 1989». Revista de Estudios Andaluces, $\mathrm{n}^{\circ}$ 22, 27-41. 